\title{
A case for extended dark matter halos in dwarf spheroidal galaxies
}

\author{
Sergey Mashchenko, H.M.P. Couchman \\ and Alison Sills \\ Department of Physics and Astronomy, McMaster University, Hamilton, \\ ON, L8S 4M1, Canada \\ email: syam,couchman,asills@physics.mcmaster.ca
}

\begin{abstract}
We explore the recently proposed idea that the Galactic dwarf spheroidal galaxies are significantly (by 2 orders of magnitude) more massive than the conventional mass estimates of $\sim 10^{7} \mathrm{M}_{\odot}$. In the larger mass case, the observed distribution of stars in these galaxies should have been entirely shaped by internal processes (formation and dynamic relaxation of stars in the potential of the dark matter halo), and not by the Galactic tidal field. We carried out numerical $n$-body simulations aimed at testing this scenario. Observed properties of three Galactic dwarf spheroidal galaxies were found to be consistent with our model. From our analysis, these dwarfs appear to be massive enough to alleviate the "missing satellites" problem of cold dark matter cosmologies.
\end{abstract}

Keywords. dark matter, early universe, galaxies: dwarf, galaxies: formation, methods: $n$-body simulations

\section{Introduction}

Cosmological cold dark matter (CDM) simulations predict that in a Milky Way sized halo there should be 1-2 orders of magnitude more subhalos than the observed number of Milky Way satellites (Moore et al. 1999). It has been suggested (Stoehr et al. 2002, Hayashi et al. 2003) that the "missing satellites" problem can be alleviated if for some reason only the most massive subhalos managed to form stars.

There are also astrophysical reasons to believe that the Galactic dwarf spheroidals (dSphs) can be much more massive than the conventional estimates of $\sim 10^{7} \mathrm{M}_{\odot}$. Odenkirchen et al. (2001) used a multi-color filtering technique to derive a high quality surface brightness map of Draco. The lack of tidal features in the outermost isodensity contours implied that Draco is more dark matter (DM) dominated in its outskirts than at its center, with a virtually unconstrained upper limit on total mass. Also, there is a contradiction between complex star formation histories and apparent absence of interstellar medium (ISM) in Galactic dSphs. Burkert \& Ruiz-Lapuente (1997) and Mashchenko et al. (2004) proposed two different scenarios to explain this puzzle. Both models assume that the ISM is still there, but the gas is fully ionized [either by supernovae Ia (Burkert \& Ruiz-Lapuente 1997) or by external ionizing radiation (Mashchenko et al. 2004)], rendering it virtually undetectable. The common requirement is that the DM halos should be massive $\left(\gtrsim 10^{9} \mathrm{M}_{\odot}\right)$ to keep the ionized gas gravitationally bound.

If dSphs are massive enough, the distribution of stars in these galaxies (including "extratidal stars" in their outskirts) should have been entirely shaped by internal processes, and not by the Galactic tidal field. We test this idea using a simple model for formation and dynamic relaxation of stars in dSphs with extended DM halos. The results of simulations are compared with the observed properties of four Galactic dSphs: Draco, Sculptor, Carina, and Fornax. 

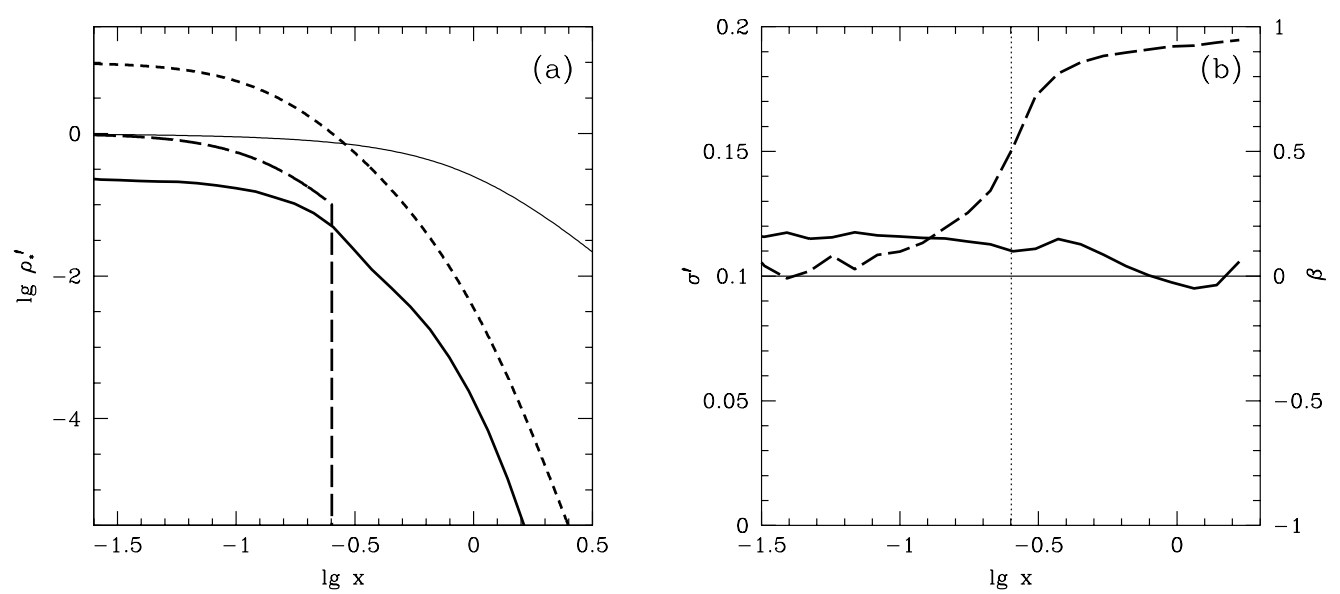

Figure 1. Radial profiles for Burkert model with $c_{g}^{\prime}=0.16, \rho_{g, 0}^{\prime}=10, \xi=0.1$, and $\lambda=0.1$. (a) Density profiles for DM (thin solid line), initial gas distribution (short-dashed line), initial stellar distribution (long-dashed line), and relaxed stellar cluster (thick solid line). (b) Total velocity dispersion $\sigma^{\prime}$ (thick solid line) and anisotropy parameter $\beta$ (long-dashed line) profiles for the relaxed stellar cluster. Vertical dotted line marks the initial stellar cluster radius. Horisontal solid line corresponds to $\beta=0$.

\section{Model}

The main assumptions in our model are as follows. Initially, gas is in hydrostatic equilibrium in the static potential of the DM halo (short-dashed line in Figure 1a). We take into account self-gravity of the gas, which is assumed to be isothermal. In a DM halo with a universal density profile, the distribution of gas depends only on two free parameters: the central gas density $\rho_{g, 0}^{\prime}$ (in units of the DM halo scaling density) and the sound speed in the gas $c_{g}^{\prime}$ (in units of the halo scaling velocity). We consider two types of DM halos: cuspy Navarro, Frenk, \& White (1997; NFW) halos and flat-cored Burkert (1995) halos. We assume that all stars in the galaxy formed in a single starburst, with a constant efficiency $\xi$ out to a radius $r_{\lambda}$ where the gas density drops down to $\lambda \rho_{g, 0}^{\prime}$. (Here $\xi$ and $\lambda$ are free parameters.) No stars are formed beyond $r_{\lambda}$ (see long-dashed line in Figure 1a). The newly born stars have one-dimensional velocity dispersion equal to the sound speed in the gas $c_{g}$ and isotropic distribution of velocity vectors. We assume that the leftover gas is instantaneously removed by stellar feedback. The stellar cluster is not in equilibrium initially, and will expand before reaching a relaxed state (solid line in Figure 1a). We study this process using $n$-body simulations.

In the relaxed stellar cluster, stellar orbits are almost isotropic at the center and strongly radially anisotropic in the outskirts, beyond the initial stellar radius $r_{\lambda}$, whereas the total velocity dispersion does not change much with radius (see Figure 1b). In projection, such a galaxy would appear to have a flat central line-of-sight velocity dispersion profile and kinematically cold outskirts, similarly to what is observed in some dSphs (Kleyna et al. 2004, Wilkinson et al. 2004).

To simulate the relaxation of stars in a static potential of the DM halo, we used a parallel $n$-body code GADGET (Springel et al. 2001). We ran around 700 models for different values of the four initial parameters $\rho_{g, 0}^{\prime}, c_{g}^{\prime}, \xi$, and $\lambda-$ for both NFW and Burkert DM halo profiles. Stars were represented by $10^{4}$ collisionless equal mass stellar particles. 

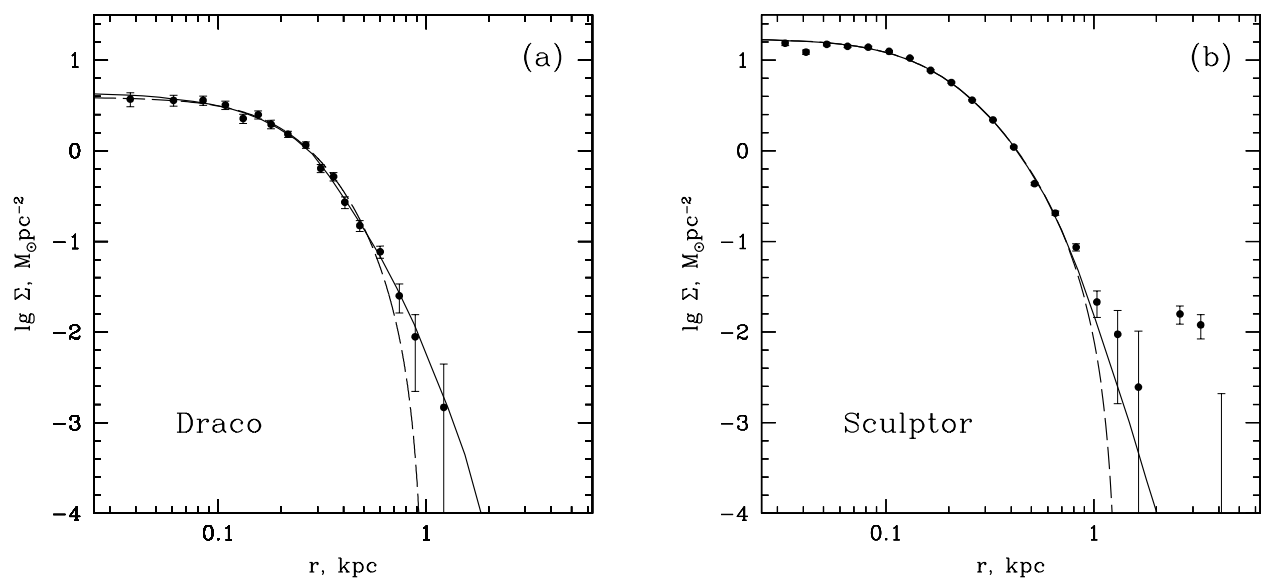

Figure 2. Surface density profiles for (a) Draco and (b) Sculptor. The observed (circles with errorbars), our best fitting model (solid lines), and the best fitting theoretical King model (dashed lines) profiles are shown.

We used three criteria to choose models which are a good match to observed dSphs. (1) The model stellar surface density profile should be a better match to the observed one than the best fitting theoretical King model profile: $\chi_{\text {model }}^{2} \lesssim \chi_{\text {King }}^{2}$. (2) The model central line-of-sight velocity dispersion value should be equal to the observed one within the observational uncertainties. (3) DM halo comoving number density in the concordance $\Lambda \mathrm{CDM}$ cosmology should be similar to or larger than the comoving number density of Local Group dSphs. (DM halo parameters are inferred after fitting the model surface density profile to the observed one.)

\section{Results}

We used star density profiles for Draco (two datasets), Sculptor, Carina, and Fornax from Odenkirchen et al. (2001), Walcher et al. (2003), and Wilkinson et al. (2004). Typically, we had 5-10 "good models" (which would satisfy all the three selection criteria from the previous section) per galaxy. The only exception was Fornax, where not a single model would satisfy the selection criteria.

In Figure 2 we show the best fitting models for Draco and Sculptor (Carina's fit is similar to that of Sculptor). For comparison, we also show the best fitting theoretical King models. As one can see, our models describe the central parts of the galaxies (out to a radius where the surface density becomes $2-2.5$ orders of magnitude lower than at the center) very well and are virtually identical to the King models. In the outer parts of the galaxies, our models significantly improve upon the King models by having less steep surface brightness profiles. In our models, the "extratidal stars" (stars located beyond the nominal King tidal radius) are gravitationally bound to dSphs and are a part of their extended stellar halos.

Our "good models" have the following parameters. Their virial masses (the masses they had when they were formed) are $\sim 10^{9} \mathrm{M}_{\odot}\left(\right.$ up to $\left.2 \times 10^{10} \mathrm{M}_{\odot}\right)$, which are large enough to alleviate the "missing satellites" problem of CDM cosmologies. They tend to have shallow inner DM density profiles (with the slope $\gamma=-0.5 \ldots 0$ ). The gas temperature 
is $\sim 10^{4} \mathrm{~K}$. The star formation efficiency is $\sim 10 \%$. The galaxy formation redshifts are $\sim 2 \ldots 6$ (lookback time $\sim 10-13$ Gyr), which is consistent with the age of the oldest stars in these galaxies.

\section{Conclusions}

Our simple star formation model for dSphs fits very well the observed properties of Draco, Sculptor, and Carina. The inferred large DM halo masses are consistent with the idea that Galactic dSphs are hosted by the most massive subhalos predicted to populate the Milky Way halo by cosmological $\Lambda \mathrm{CDM}$ simulations.

\section{Acknowledgements}

The $n$-body simulations reported in this paper were carried out at the Canadian Institute for Theoretical Astrophysics.

\section{References}

Burkert, A. 1995, ApJ (Letters) 447, L25

Burkert, A. \& Ruiz-Lapuente, P. 1997, ApJ 480, 297

Hayashi, E., Navarro, J.F., Taylor, J.E., Stadel, J. \& Quinn, T. 2003, ApJ 584, 541

Kleyna, J.T., Wilkinson, M.I., Evans, N.W. \& Gilmore, G. 2004, MNRAS 354, L66

Mashchenko, S., Carignan, C. \& Bouchard, A. 2004, MNRAS 352, 168

Moore, B., Ghigna, S., Governato, F., Lake, G., Quinn, T., Stadel, J. \& Tozzi, P. 1999, ApJ (Letters) 524, L19

Odenkirchen, M., et al. 2001, AJ 122, 2538

Springel, V., Yoshida, N. \& White, S.D.M. 2001, New Astronomy 6, 79

Stoehr F., White S.D.M., Tormen G. \& Springel V. 2002, MNRAS 335, L84

Walcher, C.J., Fried, J.W., Burkert, A. \& Klessen, R.S. 2003, A\&SA 406, 847

Wilkinson, M.I., Kleyna, J.T., Evans, N.W., Gilmore, G.F., Irwin, M.J. \& Grebel, E. K. 2004, ApJ (Letters), 611, L21

\section{Discussion}

READ: A live halo should be important. If anisotropy is introduced through mass loss to the stars then it should also be introduced to the dark matter. Also it is important to model the contraction (often not adiabatic) of the dark matter in response to the initial gas collapse. See Read \& Gilmore 2205 (MNRAS).

MASHCHENKO: I agree that this can be an important effect. We plan to test it by resimulating some of our best fitting models with live DM halos.

HENSLER: If you relax your strongly constraining assumption of instantanious gas removal, your results (stellar velocity anisotropy, density distribution, etc.) should change significantly. Did you study this effect?

MAshchenko: No, we didn't study this effect. I do not think that it will change significantly our results. E.g., even in the instantanious gas removal case the stellar cluster does not expand much (globally), so the relaxed stellar surface brightness should not depend much on the way we remove gas. The anisotropy in the outskirts will change (it will become less severe), so the outer l.o.s. velosity dispersion profile will also change. Fortunately, this data was not used in our fitting. 
Drinkwater: A few years ago I heard Bill Harris speaking about extratidal light in globular clusters. Can your type of model be applied to globulars?

Mashchenko: Yes, if globular clusters had a primordial origin with DM halos (à la Peebles). Recently we published two papers dealing with this question (Mashchenko \& Sills 2005a,b). In these papers we actually had even more consistent simulations, as our DM halos were live (non-static).

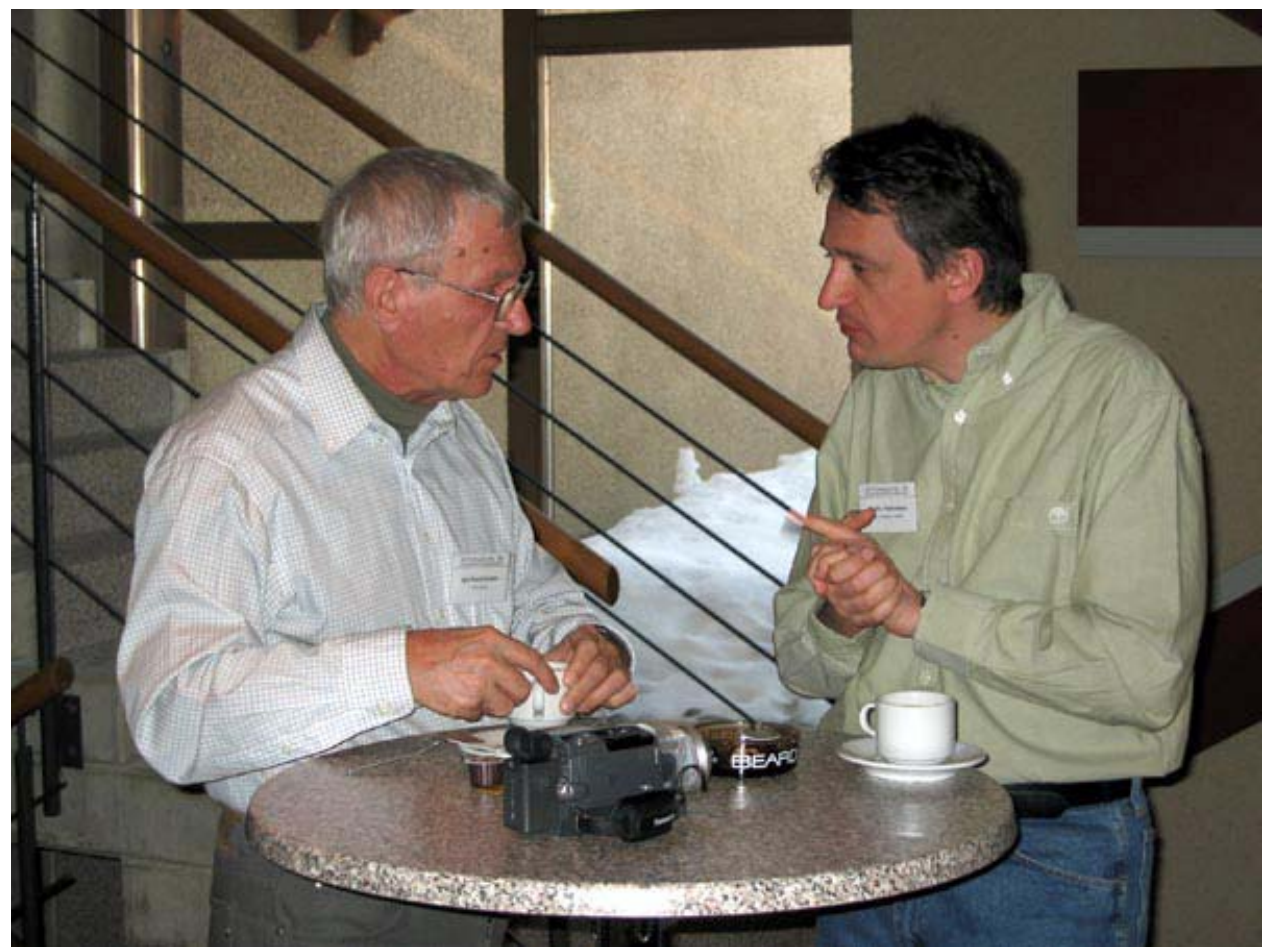

